\title{
An In-silico Approach to Explore the Possible Multifunctional Neuroprotective Efficacy of Violacein against Ischemic Stroke
}

\author{
Verma $\mathrm{S}^{1}$ and Pandey $\mathrm{AK}^{2 *}$ \\ ${ }^{1}$ Department of Biotechnology, Institute of Engineering and Technology, Dr. APJ Abdul Kalam Technical University, Lucknow, UP, India \\ ${ }^{2}$ Department of Biotechnology Engineering, Institute of Engineering and Technology, Bundelkhand University, Jhansi, UP, India
}

${ }^{*}$ Corresponding author: Pandey AK, Assistant Professor, Department of Biotechnology Engineering, Institute of Engineering and Technology, Bundelkhand University Jhansi, UP, India, Tel: +91 9125456711; E-mail: vnsanand_9@rediffmail.com

Received date: May 16, 2017; Accepted date: Jun 8, 2017; Published date: Jun 15, 2017

Copyright: (C) 2017 Verma S, et al. This is an open-access article distributed under the terms of the Creative Commons Attribution License, which permits unrestricted use, distribution, and reproduction in any medium, provided the original author and source are credited.

Citation: Verma S, Pandey AK (2017) An In-silico Approach to Explore the Possible Multifunctional Neuroprotective Efficacy of Violacein against Ischemic Stroke. J In Silico In Vitro Pharmacol Vol 3 Iss 2: 17

\section{Abstract}

Introduction: Cerebral ischemia arise due to insufficient/ interrupted blood supply to brain and is accompanied by pathologies like degradation of extracellular matrix resulting in blood brain barrier disruption, intra cranial haemorrhage, activation of astroglial cells and hence death of neurons. In addition, oxidative stress and inflammation is common to all neurodegenerative diseases enhancing the harmful consequences of diseases. Matrix Metalloproteinases (MMP) are normally involved in extracellular matrix remodelling and are regulated while injury and inflammation in tissue. Over expression of MMP2 and MMP9 results in progression of ischemic conditions by extracellular matrix degradation thus compromising the structural integrity of Blood Brain Barrier (BBB) proving them a potent targets for the disease. Violacein (source: Chromobacterium violaceum), purple pigment from microbial origin is a bisindole have high anti-inflammatory, anti-oxidant, anti-bacterial, anticancer and anti-parasitic properties. Present study is to study the effective potential of violacein against cerebral ischemia.

Methods and findings: Violacein's ADMET (Absorption, Distribution, Metabolism, Excretion, Toxicity), properties were calculated and structural inhibition of MMPs through molecular docking was evaluated. Violacein resulted six $\mathrm{H}$-bonds with $\mathrm{MMP} 2$ with high binding energy of $-11.83 \mathrm{kcal} / \mathrm{mol}$ and two $\mathrm{H}$-bonds with MMP9 with binding energy of $-9.889 \mathrm{kcal} / \mathrm{mol}$. The $\mathrm{Ki}$ values of violacein with MMP2 and MMP9 were $2.12 \mathrm{nM}$ and 56.14 $\mathrm{nM}$ respectively which proved high affinity of violacein towards the target.

Conclusion: Violacein with its high antioxidant and antiinflammatory property can be a potent all round treatment for cerebral ischemia and demands extensive research.

Keywords: Inflammation; Neuronal death; Docking analysis

\section{Abbreviations:}

ADMET: Absorption, Distribution, Metabolism, Excretion, Toxicity; BBB: Blood Brain Barrier; MMP: Matrix Metallo Proteinase

\section{Introduction}

Cerebral ischemia ranked third leading cause of death is victimizing half a million of lives every year [1]. It develops as a result of improper blood supply to brain due to haemorrhage and occlusions, which leads to inability to meet the metabolic demand and results in decreased supply of oxygen and glucose to brain cells [2]. The prolonged prevalence of such starving conditions activates intracellular and extracellular proteolytic pathways leading neuronal death and degradation of Extracellular Matrix (ECM) [3].This degradation progresses to break the basal lamina thus imparting leakage and destroying the structural integrity of blood brain barrier leading to oedema, haemorrhagic transformation, microglial cell activation, circulating inflammatory cells infiltration in brain and lastly neuronal death [4]. The composition of ECM mainly contains proteins like collagen, fibronectin, laminin and proteoglycans [5]. Matrix Metalloproteinases (MMP) are Zn dependent proteolytic enzymes responsible for the remodelling of ECM in conditions of development, inflammation and tissue injury $[6,7]$. They are activated by cytokines and are reported to hamper the integrity of ECM in ischemic conditions due to there over expression [8]. MMP2 (gelatinase A) and MMP9 (gelatinase B) plays leading role in degrading the collagen type IV protein of ECM or basal lamina thus proving themselves the most potent targets of the disease [9-11]. The increased levels of MMPs have also been 
reported in many diseases, like cardiovascular diseases, cancer, osteoarthritis, lung injury etc. causing degradation of extracellular matrix [6]. Many researchers have reported increase in expression of MMPs in cerebral ischemia [5,9-11]. Past researches have proved that MMP 9 knockout mouse models or application of MMP inhibitors and antibodies have shown protective effects and reduction in disease pathogenesis by decreasing the infraction size in ischemic brain $[9,11]$. All these findings evoke the demand to focus MMP for the contraction of disease.

Although ECM degradation by MMPs is a major pathology to deal with for combating the ischemic condition, pathologies like ionic imbalance following excitotoxicity, acidosis, periinfract depolarization, oxidative stress, inflammation, edema and apoptosis also need special attention as they contribute a lot to adverse the conditions $[12,13]$.

In diseased state increment in free radicals generation during enzymatic conversions and formation of transient pores in mitochondria activate apoptosis in cells $[14,15]$. This increase in free radicals not only cause destruction of physiology of brain by apoptotic neuronal insult but also cause, lipid peroxidation by free radicals causing membrane damage and disturb cellular processes, degradation of proteins and nucleic material in turn resulting into formation of free radical [16].This continuous cycle of free radical formation leads to oxidative stress which occurs due to the imbalance of free radical oxidant species and the anti-oxidant levels of brain [17]. The main free radicals causing oxidative stress are reactive oxygen species especially superoxide anion, hydroxyl anion, hydrogen peroxide, $\mathrm{NO}^{-}{ }_{2}$ and NO $[13,14]$. Oxidative stress causes degradative stress on mitochondria and endoplasmic reticulum causing neuronal death [17]. High amount of oxidative stress causing free radical also activate mitogen activated protein kinase pathways which too are involved in increasing inflammation and cellular damage [18]. Violacein[3-(1,2-dihydro-5-(5-hydroxy-1H-indol-3-yl)-2-oxo-3Hpyrrol-3-ilydene)-1,3-dihydro-2H-indol-2-one] is a bisindol made by condensation of two tryptophan residues (Figure 1).

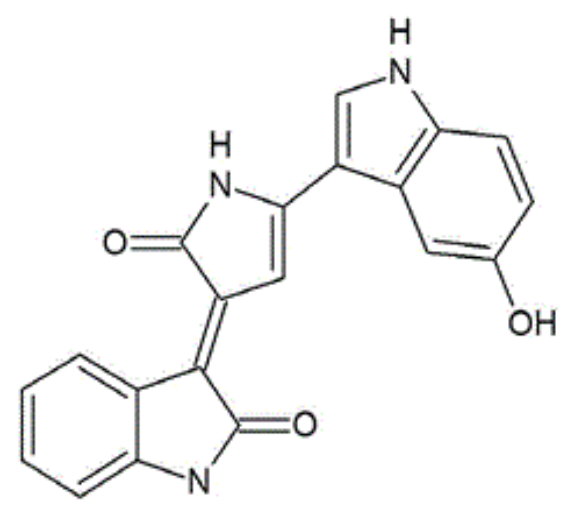

Figure 1: Structure of violacein.

It is mainly synthesized by Cromobacterium violaceum but is also found to be synthesized by Janthinobacterium lividum and Alteromonasluteo violacea under specific conditions $[19,20]$. It is a purple pigment which is insoluble in water but soluble in DMSO, methanol and ethyl acetate. It is a multifunctional compound with anti-inflammatory, anti-oxidant, anti-bacterial, anti-cancer and anti-parasitic properties [20-22]. The antioxidant property was studied by Konzen et al. for nitrogen and oxygen reactive species, 1,1-diphenyl-2-picryl hydrazyl (DPPH) and hydroxyl radical and resulted in finding the reduction of free radicals in efficient way [18]. Lipid peroxidation studies of violacein found protective effect on lipid membranes and also proved enhanced anti-oxidant property when reconstituted with membranes or liposomes as delivery systems [21,23]. Its anti-cancer property is well established in vitro due to its inhibiting potential against MMPs via cytokine inhibition in cancer cells, hence reducing tumor growth, metastasis, and angiogenesis. Also there is reduction in inflammatory chemokine secretions in turn giving anti-inflammatory effect. In a study on mice model it became evident that violacein have great potential to treat acute and chronic inflammation by supressing the cytokine production in acute inflammation and stimulating regulatory $T$ cells in chronic inflammation $[24,25]$.

Table 1: Physiological properties of violacein.

\begin{tabular}{|c|c|}
\hline Smiles & Oc1 $1 \operatorname{cc} 2 c(c 1) c(c[n H] 2) C 1=C C(=C 2 C(=O) N c 3 c 2 \operatorname{ccc} 3) C(=O) N 1$ \\
\hline Formula & $\mathrm{C}_{20} \mathrm{H}_{13} \mathrm{~N}_{3} \mathrm{O}_{3}$ \\
\hline Molecular weight & $343.34 \mathrm{~g} / \mathrm{mol}$ \\
\hline Number of heavy atoms & 26 \\
\hline Number of arom. heavy atoms & 15 \\
\hline Fraction Csp3 & 0 \\
\hline Number of rotatable bonds & 1 \\
\hline Number of $\mathrm{H}$-bond acceptors & 3 \\
\hline Number of $\mathrm{H}$-bond donors & 4 \\
\hline Molar Refractivity & 104.77 \\
\hline TPSA & $94.22 \AA^{2}$ \\
\hline
\end{tabular}


Its antioxidant property and immune modulatory power proved it a great marine nutraceutical which is effective against cancer, rheumatoid arthiritis, cardiovascular diseases and (Table 1) many neurodegenerative disorders. All these facts encouraged us to focus violacein for its role as neuroprotection in cerebral ischemia $[22,26]$.

\section{Our Hypothesis}

In the present study we hypothesize that violacein with its anti-oxidative, anti-inflammatory and MMP inhibiting properties can be considered as a potent drug for cerebral ischemia. Efforts were made to prove this hypothesis by finding the ADMET properties of violacein and by performing molecular docking, for the disclosure of any structural inhibition of MMPs, with MMP2 and MMP9 as major targets of cerebral ischemia.

\section{Methodology}

\section{ADMET properties of violacein}

The smile notation of violacein was taken from Pubchem (Pubchem CID: 9928039). Toxicity properties like LD $_{50}$ were calculated from web based tool Protox [28]. The ADME properties were measured by Swiss ADME web tool of Swiss Institute of Bioinformatics [27]. Molecular properties and bioactivity scoring of violacin were calculated by Molinspiration Chemoinformatics web based tool.

Table 2: Water solubility of violacein.

\begin{tabular}{|c|c|}
\hline Log S (ESOL) & -3.51 \\
\hline Solubility & $1.05 \mathrm{e}-01 \mathrm{mg} / \mathrm{ml} ; 3.06 \mathrm{e}-04 \mathrm{~mol} / /$ \\
\hline Class & Soluble \\
\hline Log S (Ali) & -3.48 \\
\hline Solubility & $1.14 \mathrm{e}-01 \mathrm{mg} / \mathrm{ml} ; 3.31 \mathrm{e}-04 \mathrm{~mol} / /$ \\
\hline Class & Soluble \\
\hline Log S (SILICOS-IT) & -6.45 \\
\hline Solubility & $1.21 \mathrm{e}-04 \mathrm{mg} / \mathrm{ml} ; 3.52 \mathrm{e}-07 \mathrm{~mol} / /$ \\
\hline Class & Poorly soluble \\
\hline
\end{tabular}

Table 3: Lipophilicity of violacein

\begin{tabular}{|c|c|}
\hline Log $P_{\mathrm{o} / \mathrm{w}}$ (iLOGP) & 1.4 \\
\hline Log $\mathrm{P}_{\mathrm{o} / \mathrm{w}}(\mathrm{XLOGP})$ & 1.9 \\
\hline $\log \mathrm{P}_{\mathrm{o} / \mathrm{w}}(\mathrm{WLOGP})$ & 1.8 \\
\hline $\log \mathrm{P}_{\mathrm{o} / \mathrm{w}}(\mathrm{MLOGP})$ & 1.3 \\
\hline Log $\mathrm{P}_{\mathrm{o} / \mathrm{w}}(\mathrm{SILICOS}-\mathrm{IT})$ & 3.1 \\
\hline Consensus Log $\mathrm{P}_{\mathrm{o} / \mathrm{w}}$ & 1.9 \\
\hline
\end{tabular}

Table 4: Druglikeness of violacein.

\begin{tabular}{|c|c|}
\hline Lipinski & Yes; 0 violation \\
\hline Ghose & Yes \\
\hline Veber & Yes \\
\hline Egan & Yes \\
\hline Muegge & Yes \\
\hline Bioavailability Score & 0.55 \\
\hline
\end{tabular}

Table 5: Medicinal chemistry of violacein.

\begin{tabular}{|c|c|}
\hline PAINS & 1 alert: ene_five_het_C \\
\hline Brenk & 1 alert: michael_acceptor_1 \\
\hline Leadlikeness & Yes \\
\hline Synthetic accessibility & 3.34 \\
\hline
\end{tabular}

Table 6: Pharmacokinetics of violacein.

\begin{tabular}{|c|c|}
\hline Gl absorption & High \\
\hline P-gp substrate & No \\
\hline CYP1A2 inhibitor & Yes \\
\hline CYP2C19 inhibitor & No \\
\hline CYP2C9 inhibitor & No \\
\hline CYP2D6 inhibitor & No \\
\hline CYP3A4 inhibitor & No \\
\hline Log K (skin permeation) & $-7.06 \mathrm{~cm} / \mathrm{s}$ \\
\hline
\end{tabular}

Table 7: Molinspiration bioactivity scores of violacein.

\begin{tabular}{|c|c|}
\hline GPCR ligand & -0.17 \\
\hline Ion channel modulator & -0.21 \\
\hline Kinase inhibitor & 0.52 \\
\hline Nuclear receptor ligand & 0.17 \\
\hline Protease inhibitor & -0.28 \\
\hline Enzyme inhibitor & 0.14 \\
\hline
\end{tabular}

Table 8: Result of molecular docking of violacein with MMP2 and MMP9.

\begin{tabular}{|l|l|l|l|}
\hline $\begin{array}{l}\text { Violacein } \\
\text { docked with }\end{array}$ & $\begin{array}{l}\text { Binding } \\
\text { energy }\end{array}$ & Ki value & Residues \\
\hline MMP2 & $\begin{array}{l}-11.83 \\
\mathrm{kcal} / \mathrm{mol}\end{array}$ & $2.12 \mathrm{nM}$ & $\begin{array}{l}\text { TYR223, LEU197, ALA220, } \\
\text { ALA217, GLU202 and PRO } \\
221\end{array}$ \\
\hline MMP9 & $\begin{array}{l}-9.89 \\
\mathrm{kcal} / \mathrm{mol}\end{array}$ & $56.14 \mathrm{nM}$ & TYR 420 and ARG 424 \\
\hline
\end{tabular}

\section{Docking analysis}

Docking analysis of violacein and MMPs was performed by using Autodock 4.0 software. The 3D crystal structure of MMP2 (PDB id 1QIB) and MMP9 (PDB id 1L6J) were obtained 
from RCSB (Research Collaboratory for Structural Bioinformatics) Protein Data Bank. The violacein structure was made in $A C D$ lab by using smile notation from Pubchem (Figure 1).

The PDB file of violacein was generated by using online smile translator to get the 3Dstructure for docking analysis. All the hetero atoms, including the water molecules, except $\mathrm{Zn}$ were removed from PDB files of MMP2 and MMP9. In Autodock program $\mathrm{H}$-atoms were added to MMP2 and MMP9 then nonpolar $\mathrm{H}$-atoms were merged. Lamarck genetic algorithm which is established on adaptive local search was applied as search parameter [10]. Short range Van der walls interactions and electrostatic interactions, entropy losses, hydrogen bonding were included as autodock energy based scoring function [29]. The Lamarckian genetic algorithm parameters for this study were: Number of runs $=30$, maximum number of evaluations $=2500000$, population size 150 , number of generation $=27000$, rate of crossover $=0.8$ and rate of gene mutation=0.02 [9]. Blind docking for MMP2 was performed using grid size 126,126 and 126 along $X, Y$ and $Z$ axis with spacing of $0.375 \AA$. The grid centre was set at 72.872 ( $X$ centre), 27.271 (Y centre), 20.779 (Z centre). Blind docking for MMP9 was performed with grid size 126, 126 and 126 along $X, Y$ and $Z$-axis with spacing of $0.619 \mathrm{~A}^{\circ}$ and grid centre was set at 36.89 ( $X$ centre), 38.95 ( $Y$ centre), and 34.737 ( $Z$ centre).The kollman charges added were -87.124 for MMP2 and -207.62 for MMP9. As reported by $\mathrm{Hu}$ and Shelver [29], the docking analysis was done by applying charge of $+0.95 \mathrm{e}$ on both zinc atoms for both the MMPs (MMP2 and MMP9) [30].

\section{Results}

\section{ADMET properties}

The ADMET properties and bioactivity scores of violacein are given in Tables 1-7. This compound was found to follow Lipinski's rule which provide evidence for violacein to be a potent drug [31]. The toxicity results showed that the compound belongs to toxicity class 4 with LD50 of $500 \mathrm{mg} / \mathrm{kg}$. This proved its least toxicity or no toxicity if swallowed in amounts less than $300 \mathrm{mg} / \mathrm{kg}$. Also there was no possible binding found to toxic targets suggesting it to be safe for human administration.

\section{Docking analysis}

Results of the docking study pronounced the effectiveness of violacein for treatment of cerebral ischemia. The high binding energy of $-11.83 \mathrm{kcal} / \mathrm{mol}$ with $\mathrm{Ki}$ value of $2.12 \mathrm{nM}$ for violacein-MMP2 complex gave 6 hydrogen bonds at TYR223, LEU197, ALA220, ALA217, GLU202 and PRO 221 residues (Figure 2).

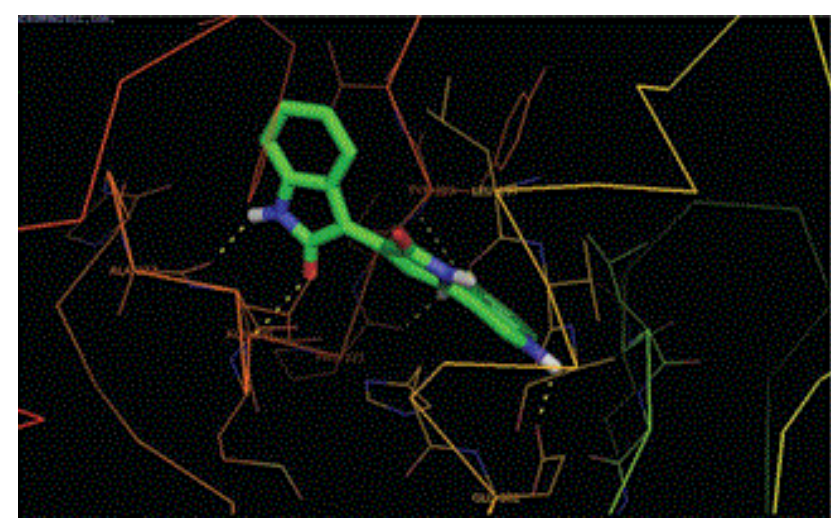

Figure 2: Violacein docked with MMP2 formed six hydrogen bonds at TYR223, LEU197, ALA220, ALA217, GLU202 and PRO 221.

Violacein also showed high binding energy when complexed with MMP9 of $-9.89 \mathrm{kcal} / \mathrm{mol}$ with 2 hydrogen bonds at TYR 420 and ARG 424 residue proving its high inhibition capability against MMPs (Figure $\mathbf{3}$ ).

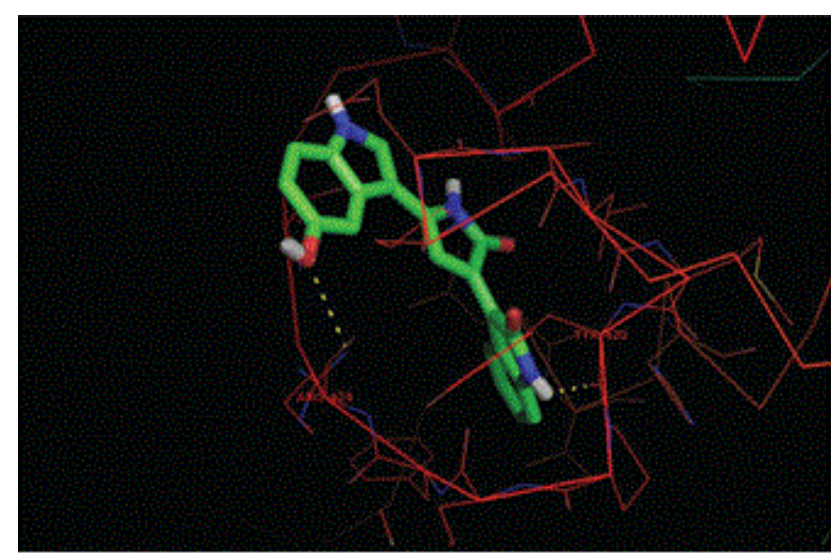

Figure 3: Violacein docked with MMP9 formed two hydrogen bonds at TYR 420 and ARG 424.

The Ki value for MMP9-violacein complex is $56.14 \mathrm{nM}$. Results of the docking study are given in Table 8.

\section{Discussion}

Cerebral occlusion and haemorrhage are the leading cause of ischemic stroke resulting into an improper blood supply to brain causing reduction in oxygen and glucose level to meet the metabolic demand [32]. As it is known that brain is the highest consumer of oxygen (about 20\%) hence any reduction may lead to harmful consequences. As the blood flow reduces to one fourth of the normal supply, the brain functions become impaired [33]. Existence of such conditions for long period leads to neuronal insult in core ischemic regions of brain continued by death of neurons in penumbra ischemic regions hence activating large number of death pathways $[3,34]$. Therapeutics to deal with this deadly condition are still 
lacking due to unknown aetiologies of neuronal insult. The knowledge about the mechanisms resulting into ischemic conversions and loss of neurons is limited and yell for more research. On the other hand the role of MMPs in progression of ischemic conditions is well established and their increase in pathological conditions is reported by many researchers $[3,5,6]$. In past study it was reported that MMP overexpression in ischemic condition is directly proportional to infraction, and reduction in infraction had been well documented when MMP inhibition was done [11]. This has encouraged promising researches to inhibit MMPs for combating ischemic conditions. As it is reported that MMP2 and MMP9 have major role in increasing the pathologies, there is great interest in finding their inhibitors for utilizing this knowledge to treat ischemia to great extent $[3,8,35-37]$.Till date many natural inhibitors have been reported for the inhibition of MMPs. Quercetin was reported by Lee et al. for its role in protection against BBB disruption and resulting edema [38]. Quercetin was also reported by Pandey et al. for its structural inhibition of MMPs. Resveratrol was reported by Pandey et al. in neuroprotection in cerebral ischemia by directly inhibiting MMPs [9]. Structural inhibition of MMPs by rutin was reported by Selvaraj et al. [36]. Violacein a microbial pigment is well accepted for its great antioxidant and anti-inflammatory potential $[19,25]$. Inhibition of MMPs is proposed by three mechanisms.

- Inhibition at transcriptional level.

- Post translational inhibition by directly attacking the active site.

- Indirect effects on endogenous activators and inhibitors that regulate MMP's activity [8,39-41].

It was reported that violacein inhibits MMP2 and MMP9 by an indirect effect thereby reducing the expression of CXCR4 which decreases cancer progression [24]. But the inhibition of MMPs by violacein for neuroprotection has not caught much attention.

Molecular docking studies are done to predict the most stable orientation of one molecule to the other when bound to form a complex [37]. Hence, in present study docking analysis of violacein with MMP2 and MMP9 was performed to get the possibility of direct active site inhibition to treat cerebral ischemia. A study by Kiyama et al. suggested that MMP9 consists of S1'cavity pocket with a floor board and MMP2 have S1'channel like cavity [41]. The S1' pocket of MMP9 consists of Asp185 to Leu188 and Pro421 to Tyr423 residues which are effective for inhibition and substrate binding [43]. The wall of S1' pocket contains side chain of Leu188, Leu397, Val398 His 401 , and Leu418 and the main chain residues Met422 to Try423 [44]. Present study of violacein with MMP9 docking found $\mathrm{H}$-bonds at Tyr 420 and Arg424 which are nearest to the active site thus proving effective inhibition.

In past studies structural inhibition of MMP2 with quercetin was found to be at residues Leu164, Ala165, Ala 217 and Ala220 with binding energy of $-9.11 \mathrm{kcal} / \mathrm{mol}$ [10]. Another study on docking analysis of rutin with MMP2 found $\mathrm{H}$ bonding at residue Glu202 with binding energy of -10.19 $\mathrm{kcal} / \mathrm{mol}$ [37]. In this study we found that violacein forms $\mathrm{H}$ bonds with Ala217, Ala220, Tyr223, Leu197, Glu202 and Pro
221 residues with a comparatively high binding energy of $-11.83 \mathrm{kcal} / \mathrm{mol}$ proving high inhibition activity against MMP2 especially because of most effective inhibition residues Ala2, Ala220 and Glu202. Docking of resveratrol with MMP9 in a study gave residues Glu402, Ala417 and Arg424 to form $\mathrm{H}$ bonds with binding energy of $-8.75 \mathrm{kcal} / \mathrm{mol}(-36.634 \mathrm{~kJ} / \mathrm{mol})$ [9]. Sarkar et al. [35] in their docking study of (-)-epicatechin-3galate and (-)-epigalocatechin-3-galate for proMMP9 inhibition found Try420 as an effective residue for inhibition with high binding energies [36]. The result obtained after docking of violacein and MMP9 complex revealed $\mathrm{H}$-bonds with binding energy of $-9.88 \mathrm{kcal} / \mathrm{mol}$ at residues Tyr 420 and $\mathrm{Arg} 424$ which were already reported as efficient residues for the inhibition hence proving our compound a better inhibitor for MMP9. Additionally the low values of $\mathrm{Ki} 2.12 \mathrm{nM}$ and $56.14 \mathrm{nM}$ for inhibition of MMP2 and MMP9 respectively in turn proved violacein a potent inhibitor. Ki value is the concentration of compound needed for effective inhibition of target. It has direct relationship with the IC50 values which is the concentration of compound required for $50 \%$ inhibition of the target enzyme. Thus lower the Ki value, higher will be the affinity of compound for its target to be inhibited [45]. In previous researches inhibition of MMP2 and MMP9 by resveratrol in a molecular docking study evaluated Ki values of $237.25 \mathrm{nM}$ and $384.27 \mathrm{nM}$ respectively which are higher than our results for violacein hence proving violacein a better inhibitor than resveratrol [9]. Also inhibition of MMP2 and MMP9 with quercetin gave Ki values $210.76 \mathrm{nM}$ and 343.46 $\mathrm{nM}$ which being greater that the Ki value of our compound provided strong support to violacein as a treatment of cerebral ischemia by inhibiting MMP2 and MMP9 with higher affinity [10].

In addition to the effective structural inhibition with high binding energies violacein is a well-established antioxidant and anti-inflammatory compound. These properties cumulatively help to reduce the pace of progression of ischemic pathologies [22]. The absorption, distribution, metabolic, excretion and toxicity analysis of violacein stood in support for its use as a drug. Hence our study conveys great scope for violacein to be an effective multifunctional drug for the treatment of cerebral ischemia. More research is anticipated to bring it in clinical trials to battle the large number of deaths due to cerebral ischemia.

\section{Conflict of Interest}

The authors declare no conflict in interests and have equal contribution.

\section{References}

1. Dugan LL, Choi DW (1999) Basic neurochemistry: molecular, cellular and medical aspects (6th edn.). Lippincott-Raven, Philadelphia.

2. Bhattacharya P, Pandey AK, Paul S, Patnaik R (2012) Aquaporin-4 in cerebral edema following ischemia/reperfusion injury: Exploration of novel therapeutic strategies. Am J Neuroprot Neuroregen 4: 90-116. 
3. Bhattacharya P, Pandey AK, Shukla SC, Paul S, Patnaik R (2013) Neuroprotection by $\mu$-calpain and matrix metalloproteinases inhibition by Piroxicam in cerebral ischemia: an in silico study. Med Chem Res 22: 5112-5129.

4. Bramlett HM, Dietrich WD (2004) Pathophysiology of cerebral ischemia and brain trauma: similarities and differences. J Cereb Blood Flow Metab 24: 133-150.

5. Lakhan SE, Kirchgessner A, Tepper D, Leonard A (2013) Matrix metalloproteinases and blood-brain barrier disruption in acute ischemic stroke. Front Neurol 4: 32

6. Dong X, Song YN, Liu WG, Guo XL (2009) MMP-9, a potential target for cerebral ischemic treatment. Curr Neuropharmacol 7: 269-275.

7. Stamenkovic I (2003) Extracellular matrix remodelling: the role of matrix metalloproteinases. J Pathol 200: 448-464.

8. Hanemaaijer R, Visser H, Koolwijk P, Sorsa T, Salo T, et al. (1998) Inhibition of MMP synthesis by doxycycline and chemically modified tetracyclines (CMTs) in human endothelial cells. Adv Dent Res 12: 114-118.

9. Pandey AK, Bhattacharya P, Shukla SC, Paul S, Patnaik R (2015) Resveratrol inhibits matrix metalloproteinases to attenuate neuronal damage in cerebral ischemia: a molecular docking study exploring possible neuroprotection. Neural Regen Res 10: 568-575.

10. Pandey AK, Verma S, Bhattacharya P, Paul S, Mishra A, et al. (2012) An in-silico strategy to explore neuroprotection by quercetin in cerebral ischemia: a novel hypothesis based on inhibition of matrix metalloproteinase (MMPs) and acid sensing ion channel 1a (ASIC1a). Medical Hypotheses 79: 76-81.

11. Romanic AM, White RF, Arleth AJ, Ohlstein EH, Barone FC (1998) Matrix metalloproteinase expression increases after cerebral focal ischemia in rats: inhibition of matrix metalloproteinase-9 reduces infarct size. Stroke 29: 1020-1030.

12. Bhattacharya P, Pandey AK, Paul S, Patnaik R, Yavagal DR (2013) Aquaporin-4 inhibition mediates piroxicam-induced neuroprotection against focal cerebral ischemia/reperfusion injury in rodents. PLoS ONE 8: e73481.

13. Chen H, Yoshioka H, Kim GS, Jung JE, Okami N, et al. (2011) Oxidative stress in ischemic brain damage: mechanisms of cell death and potential molecular targets for neuroprotection. Antioxidants \& Redox Signalling 14: 1505-1517.

14. Love S (1999) Oxidative stress in brain ischemia. Brain Pathol 9 : 119-131.

15. Sims NR, Muyderman H (2010) Mitochondria, oxidative metabolism and cell death in stroke. Biochim Biophys Acta 1802 80-91.

16. Serteser M, Ozben T, Gumuslu S, Balkan S, Balkan E (2002) Lipid peroxidation in rat brain during focal cerebral ischemia: prevention of malondialdehyde and lipid conjugated diene production by a novel antiepileptic, lamotrigine. Neurotoxicol 23: 111-119.

17. Ouyang YB, Giffard RG (2012) ER-Mitochondria Crosstalk during Cerebral Ischemia: Molecular Chaperones and ER-Mitochondrial Calcium Transfer. Int J Cell Biol 2012: 8.

18. Son Y, Cheong YK, Kim NH, Chung HT, Kang DG, et al. (2011) Mitogen-activated protein kinases and reactive oxygen species: How can ROS activate MAPK pathways? J Signal Transduct 2011: 792639.
19. Konzen M, De Marco D, Cordova CA, Vieira TO, Antonio RV, et al. (2006) Antioxidant properties of violacein: possible relation on its biological function. Bioorg Med Chem 14: 8307-8313.

20. Duran N, Justo GZ, Ferreira CV, Melo PS, Cordi L, et al. (2007) Violacein: properties and biological activities. Appl Biochem Biotechnol 48: 127-133.

21. Andrighetti-Fröhner C, Antonio R, Creczynski-Pasa T, Barardi C, Simões C (2003) Cytotoxicity and potential antiviral evaluation of violacein produced by Chromobacteriumviolaceum. Mem Inst Oswaldo Cruz 98: 843-848.

22. Lin SK (2013) Marine Nutraceuticals: Prospects and Perspectives. By Se-Kwon Kim, CRC Press, 2013; 464 Pages. Price £108.00, ISBN 978-1-4665-1351-8. Mar. Drugs 11: 1300-1303.

23. Antonisamy $P$, Kannan $P$, Aravinthan A, Duraipandiyan V, ValanArasu M, et al. (2014) Gastroprotective activity of violacein isolated from Chromobacterium violaceum on indomethacininduced gastric lesions in rats: Investigation of potential mechanisms of action. Sci World J 2014: 10.

24. Platt D, Amara S, Mehta T, Vercuyssee K, Myles EL, et al. (2014) Violacein inhibits matrix metalloproteinase mediated CXCR4 expression: potential anti-tumor effect in cancer invasion and metastasis. Biochem Biophys Res Commun 455: 107-112.

25. Verinaud L, Lopes SC, Prado IC, Zanucoli F, da Costa AT, et al. (2015) Violacein Treatment Modulates Acute and Chronic Inflammation through the Suppression of Cytokine Production and Induction of Regulatory T Cells. PloS ONE 10: e0125409.

26. Soliev AB, Hosokawa K, Enomoto K (2011) Bioactive pigments from marine bacteria: applications and physiological roles. J Evid Based Compl Altern Med 2011: 17.

27. Daina A, Michielin O, Zoete V (2017) Swiss ADME: a free web tool to evaluate pharmacokinetics, drug-likeness and medicinal chemistry friendliness of small molecules. Sci Rep 7: 42717.

28. Drwal MN, Banerjee P, Dunkel M, Wettig MR, Preissner R (2014) ProTox: a web server for the in silico prediction of rodent oral toxicity. Nucleic Acids Res 42: W53-W58.

29. Morris GM, Goodsell DS, Halliday RS, Huey R, Hart WE, et al. (1998) Automated docking using a Lamarckian genetic algorithm and an empirical binding free energy function. J Comput Chem 19: 1639-1662.

30. Hu X, Shelver WH (2003) Docking studies of matrix metalloproteinase inhibitors: zinc parameter optimization to improve the binding free energy prediction. J Mol Graph Model 22: $115-126$.

31. Lipinski CA, Lombardo F, Dominy BW, Feeney PJ (2001) Experimental and computational approaches to estimate solubility and permeability in drug discovery and development settings. Adv Drug Deliv Rev 46: 3-26.

32. Ovbiagele B, Goldstein LB, Higashida RT, Howard VJ, Johnston SC, et al. (2013) Forecasting the future of stroke in the United States: a policy statement from the American Heart Association and American Stroke Association. Stroke 44: 2361-2375.

33. Lee JM, Grabb MC, Zipfel GJ, Choi DW (2000) Brain tissue responses to ischemia. J Clin Invest 106: 723-731.

34. Kunz A, ladecola C (2009) Cerebral vascular dysregulation in the ischemic brain. Handb Clin Neurol 92: 283-305.

35. Shineman DW, Fillit HM (2009) Meeting the unique challenges of drug discovery for neurodegenerative diseases. BMC Neurol 9: I1. 
36. Sarkar J, Nandy SK, Chowdhury A, Chakraborti T, Chakraborti S (2016) Inhibition of MMP-9 by green tea catechins and prediction of their interaction by molecular docking analysis. Biomed Pharmacother 84: 340-347.

37. Selvaraj G, Kaliamurthi S, Thiruganasambandam R (2016) Molecular docking studies of rutin on matrix metalloproteinase. Insights Biomed 1: 1.

38. Lee JK, Kwak HJ, Piao MS, Jang JW, Kim SH, et al. (2010) Quercetin reduces theelevated matrix metalloproteinases-9 level and improves functional outcomeafter cerebral focal ischemia in rats. Acta Neurochir 153: 1321-1329.

39. Courtman DW, Franco CD, Meng Q, Bendeck MP (2004) Inward remodeling of the rabbit aorta is blocked by the matrix metalloproteinase inhibitor doxycycline. J Vasc Res 41: 157-165.

40. Golub LM, Lee H, Ryan ME, Giannobile WV, Payne J, et al. (1998) Tetracyclines inhibit connective tissue breakdown by multiple non-antimicrobial mechanisms. Adv Dent Res 12: 12-26.
41. Jonat C, Chung FZ, Baragi VM (1996) Transcriptional downregulation of stromelysin by tetracycline. J Cell Biochem 60: 341-347.

42. Kiyama R, Tamura $\mathrm{Y}$, Watanabe F, Tsuzuki $\mathrm{H}$, Ohtani $\mathrm{M}$, et al. (1999) Homology modeling of gelatinase catalytic domains and docking simulations of novelsulfonamide inhibitors. J Med Chem 42: 1723-1738.

43. Tondon A, Sinha S (2011) Structural insights into the binding of MMP9 inhibitors. Bioinformation 5: 310-304.

44. Tochowicz A, Maskos K, Huber R, Oltenfreiter R, Dive V, et al. (2007) Crystal structures of MMP-9 complexes with five inhibitors: contribution of the flexible Arg424 side-chain to selectivity. J Mol Biol 371: 989-1006.

45. Hossain MM, Roy PK, Mosnaz ATMJ, Shakil SK, Hasan MM, et al. (2015) Structural analysis and molecular docking of potential ligands with chorismate synthase of Listeria monocytogenes: A novel antibacterial drug target. IJBB 52: 45-59. 